\title{
EDITORIAL
}

\section{Integration of energy, ecology and environment}

\author{
Guest Editor: Bin CHEN (凶)
}

State Key Laboratory of Water Environment Simulation, School of Environment, Beijing Normal University, Beijing 100875, China

(C) Higher Education Press and Springer-Verlag Berlin Heidelberg 2014

\section{Background}

The rising concern about global energy crisis and its impact on environment implies a transition from the current development paradigm to a sustainable and desirable one, which remains a significant challenge for scientists, industries and governments to make reasonable energy and environmental policies to save energy and reduce environmental impact as well as carbon emission during the managing and controlling process at each level of the concerned systems. A special issue of Frontiers of Earth Science is therefore established to be dedicated to the integration of energy, ecology and environmental problems.

We invited investigators from universities, institutes and governments to contribute original research articles as well as review articles that can advance our understanding of the interactions between the energy system and biophysical environment in the context of ecology, economic and society at local, national and international levels.

\section{Papers in this issue}

These papers cover a variety of overlapping and transdisciplinary areas, including integration of energy and ecology, ecology and environment, and environment and energy issues.

\subsection{Integration of energy and ecology issues}

Voinov and Filatova (P3-P17) presented an explorative model that demonstrates that a smooth transition from an oilbased economy to alternative energy sources is possible only if it is started well in advance while fossil resources are still abundant. Two transparent and flexible methods of pricing were also suggested to eliminate price externalities on energy resources, which may help stimulate less consumption and can smooth the transition to renewable energy.

Chen S H et al. (P18-P31) studied the response of energy partition to land use using the Noah model. The trend of the response of sensible heat flux (SHF) and latent heat flux (LHF) to the land use change (LUC) over three typical ecological zones in China is originally quantified using the Noah results for foreknowing the partition status of the net radiant energy (NRE) in land-atmosphere interaction.

To present a systematic evaluation framework for urban ecosystem, Ji et al. (P32-P43) constructed new evaluation indicators, based on the concepts of embodied energy and embodied carbon emissions, which take both the immediate and indirect effects of energy consumption and emissions into account. With a case study of Beijing ecosystem, conventional evaluation indicators were compared with the newly constructed ones. Results show that the energy consumption and emissions of urban industries are described better by the new indicators, which may provide useful information for urban ecosystem structure optimization.

Considering that objective expression of ecosystems is an urgent concern in sustainable ecosystem management, Nguyen et al. (P44-P57) combined the community information measurements such as Shannon index and exergy and their variance formulas to assess the ecological integrity and thereby provided extra scopes of structural and functional properties in communities in response to natural variability and anthropogenic disturbance. The proposed method was also recommended as a suitable reference system for assessment of the health of

Received December 17, 2013

E-mail: chenb@bnu.edu.cn 
aquatic ecosystems exposed to environmental disturbances.

Zhang Q Z et al. (P58-P63) characterized two indigenous bacteria of petroleum contaminated soil to utilize diesel fuel as the sole carbon and energy sources, which can be identified to be better than exotic bacterial strains and chemicals for bioremediation of petroleum contaminated sites.

\subsection{Integration of ecology and environment issues}

Chen Q W et al. (P64-P69) used set pair analysis to study the dry and wet compensation characteristics of water resource parameters, of which the fuzzy membership and grey correlation degree are adopted to test the result of set pair analysis.

Dong et al. (P70-P80) conducted a coupled planning of water resources, in which agricultural land use was undertaken through the development of an inexact-stochastic programming approach. Interval linear programming (ILP) and chance-constraint programming (CCP) approaches were integrated into a general framework to tackle uncertainties and complexities expressed as intervals and probabilistic distributions, with a case study of typical regional water resource system in an agricultural watershed. The Dongjiang River is taken as an example, with parameters include precipitation and mean discharge from different hydrological stations being analyzed.

Xu et al. (P81-P92) developed an integrated model system by coupling a simple global socio-economic model and regional spatial allocation model, and applied it to the land use system in China. A series of simulations were conducted to demonstrate how the integrated model link global socioeconomic factors with regional land use changes in China.

Yin et al. (P93-P103) analyzed the impacts of climate change and human activities on grassland ecosystem at different spatial and temporal scales. The regression and residual analysis were used based on the data from remote sensing data and meteorological stations to quantify the effects of human activities and climatic factors.

A comprehensive review of the international methods used to prioritize chemicals for environmental management was conducted by Yu et al. (P104-P114), based on which an appropriate screening method was developed to account for the unique characteristics of chemical use within China. The proposed classification method may help decision-makers to prioritize and identify the chemicals with the highest environmental risk, thereby providing a basis for improving chemical management.

Yuan et al. (P115-P122) presented an analytical study of a pulsed volatile contaminant emission into a freesurface wetland flow. An eco-model is given for environmental contaminant transport under the combined action of advection, mass dispersion, apparent reaction, and volatilization at the free water surface.

\subsection{Integration of environment and energy issues}

Chen $C$ et al. (P123-P130) introduced the life cycle assessment to calculate the environmental impact loads of different types of energy resources used in urban regions. The proposed evaluation method is also applied to Beijing, revealing that photochemical oxidant formation and acidification are the primary impact factors in the life cycle of all energy resources.

Chen D et al. (P131-P141) used emergy to evaluate a pumping irrigation water production system in China. A framework for emergy evaluation of the significance of irrigation water and its production process was developed.

Guan et al. (P142-P149) provided a concrete study to understand the effects of operation on biofilm morphology and microstructure and degradation efficiency for the disposal of sulfur dioxide produced by coal-fired power plants. A flat-panel reactor-membrane bioreactor (MBR) with a composite membrane consisting of a dense layer and a support layer was designed, and the membrane bioreactors inoculated with Thiobacillus ferrooxidans were further synthetically analyzed for the removal of sulfur dioxide.

Embodied exergy-based analysis as an effective approach to achieve a unified and scientific assessment is devised by Meng et al. (P150-P162) to assess the energy and resource consumption of buildings. A systematic accounting of the landmark buildings in E-town, Beijing is performed, on the basis of raw project data in the Bill of Quantities and the most recent embodied exergy intensities for the Chinese economy in 2007 with 135 industrial sectors.

Zhang B et al. (P163-P180) also presented an interprovincial input-output embodiment analysis of China's regional $\mathrm{CH}_{4}$ emissions in 2007 based on the multi-regional input-output table and relevant $\mathrm{CH}_{4}$ emissions data. Comprehensive mitigation measures are suggested to achieve regional $\mathrm{CH}_{4}$ emission mitigation under consideration of regional transfer of emission responsibility.

The Guest Editor would like to express his high appreciation to the authors and reviewers for their great contribution to this special issue. 